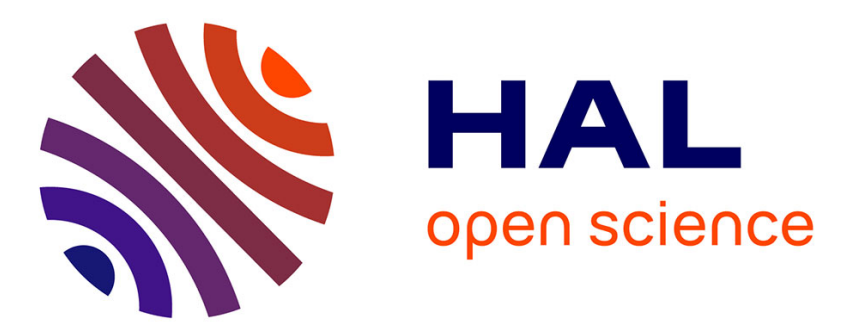

\title{
Gouverner les ouvriers dans le Japon d'avant-guerre: la modernisation comme technique de gestion des populations
}

\author{
Bernard Thomann
}

\section{- To cite this version:}

Bernard Thomann. Gouverner les ouvriers dans le Japon d'avant-guerre: la modernisation comme technique de gestion des populations. Ebisu - Études Japonaises , 2010, 44, pp.67 - 85. 10.3406/ebisu.2010.1779 . hal-03222490

\section{HAL Id: hal-03222490 \\ https://hal.science/hal-03222490}

Submitted on 10 May 2021

HAL is a multi-disciplinary open access archive for the deposit and dissemination of scientific research documents, whether they are published or not. The documents may come from teaching and research institutions in France or abroad, or from public or private research centers.
L'archive ouverte pluridisciplinaire HAL, est destinée au dépôt et à la diffusion de documents scientifiques de niveau recherche, publiés ou non, émanant des établissements d'enseignement et de recherche français ou étrangers, des laboratoires publics ou privés. 


\section{Citer ce document / Cite this document :}

Thomann Bernard. Gouverner les ouvriers dans le Japon d'avant-guerre : la modernisation comme technique de gestion des populations. In: Ebisu, $n^{\circ} 44,2010$. La modernisation du Japon revisitée. Que reste-t-il de l'approche moderniste ? [Sous la responsabilité éditoriale de Christine Lévy] pp. 67-85;

doi : https://doi.org/10.3406/ebisu.2010.1779

https://www.persee.fr/doc/ebisu_1340-3656_2010_num_44_1_1779

Fichier pdf généré le 26/03/2019 


\section{Gouverner les ouvriers dans le Japon d'avant-guerre La modernisation comme technique de gestion des populations}

\section{Bernard THOMANN}

INALCO

La question de la gestion des populations ouvrières dans le Japon d'avantguerre, dans la littérature japonaise en sciences sociales, a surtout été traitée par les spécialistes de la politique sociale ${ }^{1}$. La politique sociale s'est imposée, dès la période d'avant-guerre, comme une discipline majeure, par l'abondance de ses publications, mais aussi parce que nombre de ses représentants ont été très largement associés à l'élaboration des politiques elles-mêmes. Ils ont en effet exercé à l'université impériale de Tokyo où la plupart des hauts fonctionnaires étaient formés, mais ont aussi exercé dans un certain nombre d'associations, de centres de recherches, où ils côtoyaient les hauts fonctionnaires, du ministère de l'Intérieur en particulier.

Pourtant, malgré la place éminente de la politique sociale dans le Japon d'avant 1945, les études historiques, produites au cours de la période de l'aprèsguerre, et portant sur les conditions du développement économique et social du Japon, ont eu tendance à négliger cette dimension et ont souvent échoué à la traiter dans une problématique de modernisation. C'est tout d'abord vrai des recherches en japonologie produites par des Occidentaux qui ont eu tendance à se focaliser sur d'autres dimensions, qui étaient considérées comme des secteurs d'excellence du modèle de gouvernance japonais. On peut citer

1. Si la politique sociale (shakai seisaku 社会政策) constitue la littérature la plus importante, nous considérons néanmoins que ce que nous entendons par "gestion des populations ouvrières " recouvre aussi d'autres domaines, ayant eu tendance à être traités de manière séparée dans les sciences sociales japonaises, tels que le travail social, la gestion des ressources humaines dans l'entreprise, les politiques économiques ou bien encore les politiques démographiques. 
en particulier la politique industrielle ou le système de gestion des entreprises souvent considérés comme les domaines les plus dignes d'intérêt dans le processus de développement économique et social du Japon au $\mathrm{xx}^{\mathrm{e}}$ siècle.

En ce qui concerne les recherches en sciences sociales japonaises, la question est plus complexe. Le courant marxiste qui domine au cours de l'aprèsguerre a, d'un côté, considéré la politique sociale, la politique industrielle et les systèmes de gestion des entreprises comme un tout au sein de la catégorie plus vaste des forces et des rapports de production capitalistes. Néanmoins, d'un autre côté, cette littérature marxiste a eu aussi tendance à considérer la politique sociale à travers une grille de lecture tendant à montrer son arriération plutôt qu'à éclairer sur sa fonction dans le processus de modernisation du pays. Nous essayerons dans un premier temps d'en examiner les raisons, puis nous verrons comment, et sous quelles conditions, on peut réintroduire cette problématique de la modernisation dans l'étude de l'histoire de la gestion des populations ouvrières.

\section{Le débat Ōkōchi-Kazahaya :}

de la politique sociale comme instrument de modernisation à la thèse du sous-développement du système socio-économique japonais

Si l'histoire de la gestion des populations ouvrières a eu tendance à ne pas être traitée dans une problématique de modernisation, cela est dû, tout d'abord et sur un plan très général, à la place dominante du kōzaha 講座派 ${ }^{2}$, qui commence à articuler ses thèses dans les années 1930 et devient un courant de pensée dominant après la guerre. Cette école de pensée, influencée au départ par les thèses du Kominterm de 1932 sur l'état de développement économique, social et politique du Japon, tendait en effet à ne voir dans les politiques du gouvernement japonais d'avant-guerre que des signes de sousdéveloppement et de retard dans la typologie du développement marxiste, la

2. Ce mouvement historiographique doit son nom à une série d'ouvrages parus sous le titre Nihon shihonshugi hattatsushi kōza 日本資本主義発達史講座, Tokyo, Iwanami Shoten 岩波書店, 1932-33. Une des raisons pour lesquelles cette publication a particulièrement marqué l'historiographie japonaise est qu'il s'agissait de la première tentative aussi systématique de traiter des conditions du développement économique japonais contemporain. 
preuve que la restauration Meiji n'avait pas véritablement été une révolution bourgeoise et n'avait pas permis au pays de véritablement dépasser le stade de la féodalité. Cependant, ce biais de l'historiographie japonaise est dû aussi à l'importance et à la forme que prit le débat entre les deux représentants les plus éminents de l'étude de la politique sociale : Ōkōchi Kazuo 大河内一男 et Kazahaya Yasoji 風早八十二.

Juste avant la guerre du Pacifique, Ōkōchi Kazuo avait essayé de faire de la politique sociale une discipline scientifique liée à la science économique, capable de véritablement jouer un rôle dans la modernisation du système socioéconomique japonais. Il avait pour cela critiqué l'approche qui avait, selon lui, caractérisé la réforme sociale jusqu’alors. Il avait commencé par articuler cette critique autour du cas allemand, ayant conscience que c'était la politique sociale allemande qui avait le plus largement influencé la politique sociale japonaise. Pour lui, la politique sociale allemande, dans sa forme la plus classique et primitive, celle de la " théorie de la coopération " (kyōchō riron 協 調理論), apparaît en effet d'abord sous la couleur " morale " des " socialistes de chaire" (ködan shakaishugisha 講壇社会主義者). Ceux-ci, hantés par la question de l'opposition entre les intérêts de classe et à la recherche d'une approche plus scientifique, vont, en se reposant sur des considérations avant tout humanitaires, réduire la politique sociale à une politique de partage, une " politique de partage moralisée " (dōtokuteki bunpai seisaku 道徳的分配政策) (Ōkōchi 1939 : 80). Cependant, pour Ōkōchi, il ne s'agissait pas là d'une analyse véritablement scientifique de la lutte des classes, il n'y avait que l'expression primaire des socialistes de chaire reposant sur un "sens commun humanitaire " (jöshikiteki na jindōshugi 常識的な人道主義). En effet, toujours selon Ōkōchi, les réformateurs sociaux allemands, ressentant une indignation morale devant les injustices et les nuisances sociales qui se présentaient à leurs yeux avaient voulu retrouver des fondements éthiques et religieux à l'action sociale, mais ils n'avaient pas eu l'audace d'aller jusqu'à véritablement analyser les règles qui se cachaient derrière le développement économique, les fondements des nuisances sociales et des injustices qui provoquaient leur indignation. Même si une telle réflexion était amorcée, elle restait pour Ōkōchi à un stade enfantin. Ils se contentaient de compenser l'immaturité de leur réflexion en invoquant des principes idéaux (Ōkōchi 1939 : 93). 
Concernant la politique sociale japonaise proprement dite, Ōkōchi voulait étudier la signification de la Loi sur les usines (Köjōhō 工場法) de $1911^{3}$ dans le système socio-économique de l'époque. Il était d'une part influencé par le premier tome du Capital où Marx analysait la loi sur les usines britanniques et était, d'autre part, insatisfait de l'explication qui était souvent donnée des lois de protection des ouvriers comme étant avant tout des mesures d'assistance sociale d'État de nature éthique. Il estimait, à la suite de Marx, qu'il s'agissait en réalité d'une mesure rationnelle pour assurer la reproduction de la main d'œuvre (Ōkōchi 1968 : 415-427). D'autre part, même si cette politique de législation sociale avait été effectivement comprise comme une mesure contre les dommages causés par l'industrialisation capitaliste, elle avait été aussi vue comme une intervention de type non économique dans les relations du travail capitalistes. Or Ōkōchi estimait au contraire qu'il fallait voir cette politique sociale comme une partie de la politique économique capitalistique (shihonseiteki shakai seisaku 資本制的社会政策), comme un moyen de réaliser le développement sain d'une économie capitaliste et d'assurer le maintien et le renforcement de ce système. La politique sociale était l'expression consciente de la préservation d'un système et d'un processus de développement capitaliste dans ses dimensions aussi bien sociales qu'économiques (Ōkōchi 1940a : 239). Ainsi, si la législation sociale ou le paternalisme d'entreprise encouragé par l'État ne s'attaquaient que superficiellement aux injustices et aux dégâts sociaux générés par l'industrialisation, sans s'attaquer réellement aux causes profondes de ces injustices et de ces dégâts, c'est que la racine de ces dernières n'était pas tant d'ordre moral, que d'ordre économique. Elle résidait principalement dans le prix trop faible qui était alloué à la force de travail. Ainsi, le meilleur moyen de corriger ces injustices n'était pas tant de recourir à des mesures humanitaires, n'ayant pour rationalité que le respect de la personne humaine, que de considérer jusqu'au bout le travailleur pour ce qu'il était au sein des rapports de production - une force de travail - et de produire des mesures de réforme économique capables d'augmenter le prix qui lui était accordé. Quand le pays plongea encore davantage dans la guerre à la fin des années 1930, Ōkōchi, fort de sa théorie sur la force de travail fut très large-

3. Votée en 1911 et entrée en vigueur en 1916, la Loi sur les usines $(K o \bar{j} j \bar{h} h \overline{)})$ interdit le travail des enfants de moins de 12 ans, réglemente le temps de travail pour les femmes et les enfants de moins de 15 ans, met en place un corps d'inspecteurs du travail et reconnait la responsabilité de l'employeur dans les accidents du travail. 
ment impliqué dans la politique de mobilisation générale via son appartenance à la Shōwa kenkyūkai 昭和研究会, mais aussi son rôle d'expert pour un certain nombre d'autres commissions chargées de la politique économique et sociale, en particulier la Commission centrale sur les prix (Chūō bukka kyōryoku kaigi 中央物価協力会議) qui avait notamment en charge la politique des salaires. En effet, pour lui, la guerre ne devait pas avoir pour conséquence une mise entre parenthèses de la politique sociale, mais devait au contraire être l'occasion de la développer dans des domaines où cela était impossible jusqu'alors. Elle jouait en quelque sorte comme un accélérateur de l'histoire dans le domaine de la modernisation des structures économiques et sociales.

La relation entre la guerre et la politique sociale était à deux niveaux : la seconde était la conséquence logique de la première dans le sens où elle devait en corriger les effets déstabilisateurs sur le plan social. Mais elle devait aussi être l'occasion de vaincre les oppositions traditionnelles, patronale en particulier, et de rendre la politique sociale indispensable à long terme (Ōkōchi 1940b : 4). En ce qui concerne le premier point, une politique sociale était essentielle pour garder les hommes en bonne santé dans le but d'avoir de bons soldats, mais aussi des travailleurs dans les usines d'armement en pleine possession de leurs moyens. Si la guerre durait, la main d'œuvre venait à manquer, il fallait alors se tourner vers les femmes et les enfants, une amélioration des conditions de travail devenait encore plus nécessaire, d'autant plus que c'était le futur de la nation qui était en jeu (Ōkōchi 1940b : 33, 129-131). La première guerre mondiale avait d'ailleurs montré, qu'une guerre, c'était aussi une guerre économique et qu'une politique de production efficace ne pouvait se passer d'une politique sociale assurant la protection et la reproduction d'une main d'œuvre pensée comme une force de production (Ōkōchi 1940b : 157).

Toujours est-il qu'Ōkōchi fut surtout impliqué dans des entreprises de réforme des mécanismes de financement de la production et des systèmes de salaire ${ }^{4}$ qui avaient des objectifs profondément ambigus. Ces réformes eurent, d'une part, une fonction de mobilisation des ressources - orienter les ressources financières vers la production d'armement, préserver ou améliorer

4. En 1944 fut par exemple mis sur pied le Senji seikatsu sōdanjo 戦時生活相談所, organisation affiliée au gouvernement, dont un des objectifs était la mise en place d'un système de salaire mieux adapté aux besoins effectifs des salariés. Ōkōchi y fut un membre clé aux côté d'Andō Masakichi 安藤政吉 ou encore de Sugimoto Eiichi 杉本栄一 de l'université Hitotsubashi 一橋大学 (Ōkōchi 1970 : 179). 
les conditions de travail pour maintenir la condition physique de la main d'œuvre pour la rendre plus productive et assurer sa reproduction. Mais les réformes eurent aussi pour objectif, suivant là l'inspiration d'Ōkōchi, de mettre en place des rapports de production plus modernes permettant l'allocation d'un prix plus élevé à la force de travail.

Kazahaya Yasoji qui était l'autre grand spécialiste de la politique sociale dans ces années-là, membre également de la Shōwa kenkyūkai, reprocha cependant à cette approche de voir le travailleur comme un élément purement passif dans ce processus de modernisation et de considérer le mouvement ouvrier comme un facteur peu décisif. L'existence des politiques sociales dans les sociétés industrialisées était en effet, selon Ōkōchi, surtout liée au souci du capitalisme d'assurer la reproduction de ses forces de travail et ainsi sa propre reproduction. Dans son ouvrage majeur publié en 1937 - Histoire de la politique sociale au Japon (Nihon shakai seisaku shi 日本社会政策史) Kazahaya estimait lui que la politique sociale était à la rencontre de la politique économique et de la politique elle-même et non un sous-ensemble de la science économique. En effet, selon lui, l'existence de la lutte des classes et du danger qu'elle représentait pour la société capitaliste était un pré-requis pour la naissance d'une politique sociale dont une fonction était ainsi d'assurer une certaine redistribution des richesses (Kazahaya 1937).

Après la défaite, Kazahaya, auréolé de plusieurs arrestations, intensifia ses critiques à l'encontre d'Ōkōchi auquel il reprochait d'avoir évacué la question de la lutte des classes de sa théorie de la politique sociale alors même qu'il se prétendait marxiste et qu'il disait avoir participé à ce titre au front populaire contre le fascisme ${ }^{5}$. Ōkōchi tenta de démontrer le bien-fondé de sa thèse au sein de l'appareil théorique marxiste, mais, dans le même temps, ses problématiques de recherche se déplacèrent et il devint surtout un des historiens les plus reconnus du travail et du mouvement ouvrier ${ }^{6}$. Il tenta en particulier d'isoler les facteurs de sous-développement du mouvement ouvrier avant la guerre. C'est ainsi qu'il devint l'auteur de la théorie du travailleur migrant (dekasegi rōdōsha 出稼ぎ労働者) d'origine rurale qui aurait retardé la naissance d'une conscience de classe au sein de la classe ouvrière (Ōkōchi 1970). Cela eut des conséquences pour ce qui nous intéresse ici : alors qu’il était avant

5. Kazahaya faisait référence au front populaire décrété par le Kominterm lors de son congrès de 1935 .

6. Pour un compte rendu détaillé du débat voir Kishimoto (1950). 
la guerre le principal défenseur de la thèse d'une politique sociale, facteur de modernisation économique et sociale, il apportait, après la guerre, son prestige académique à la thèse d'un sous-développement du système socioéconomique japonais de l'avant-guerre. Pendant le même temps, Kazahaya, suivant sa ligne théorique, développa la thèse selon laquelle, en raison de la politique répressive de l'État à l'égard du mouvement ouvrier, et comme l'existence de ce dernier en était un des pré-requis, la politique sociale d'avantguerre avait avant tout été caractérisée par son arriération.

Ainsi, certains efforts de la période d'avant-guerre pour développer une politique sociale, loin d'être considérés comme des politiques modernisatrices, furent ignorés ou même interprétés comme des manifestations du caractère réactionnaire et du sous-développement de l'État impérial. Un exemple particulièrement parlant est celui de la Kyōchōkai 協調会 (la Société pour la coopération). Elle fut, comme des études récentes l'ont montré, créée pour répondre aux défis posés par l'adhésion du Japon à l'Organisation internationale du Travail en 1919 et constitua une des innovations institutionnelles les plus significatives de l'avant-guerre dans le développement d'une politique sociale présentant une alternative réformiste à la révolution prolétarienne, alors que, jusqu'à il y a une dizaine d'années elle était uniquement présentée comme un instrument de répression du mouvement ouvrier. Cette littérature marxiste a bien évidemment souligné avec raison tout l'appareil administratif, policier et militaire dont la société civile fut victime au cours de la période d'avant-guerre, cependant, elle a surtout eu tendance, comme nous l'avons déjà souligné, à y voir des manifestations de sous-développement alors qu'il faut sans doute lui donner un sens plus complexe dans le processus de modernisation.

\section{Réintroduire la problématique de la modernisation}

\section{dans l'histoire des politiques de gestion des populations ouvrières}

Premièrement, pour redonner à la politique sociale japonaise la place qu'elle mérite, et aux politiques de gestion des populations ouvrières leur juste signification dans le processus de modernisation économique et sociale du Japon, il nous semble important de ne pas les réduire à un processus d'imposition par le haut - par l'État - de mesures de contrôle social. En effet, une des causes de la réfutation de l'existence d'une politique sociale, facteur de modernisation avant la guerre, a été la thèse soutenue par Kazahaya comme on l'a vu, et plus 
largement par l'historiographie marxiste dans sa majorité, d'une société civile écrasée par un "capital monopolistique d'État».

Il ne s'agit bien évidemment pas de nier la sévère répression dont fut victime le mouvement ouvrier, mais de montrer que la société civile, sous des formes diverses, eut quand même des espaces d'expression d'où elle put influencer la formation des politiques gouvernementales. On peut d'ailleurs revenir ici à une réflexion de Michel Foucault sur la nécessité de ne pas avoir une vision univoque de la forme du pouvoir. Dans ses cours de l'année 1977-1978 au Collège de France, il rappelle qu'une des sources de fécondité de l'histoire de l'État comme manière de faire et de penser est de considérer qu'il n'y a pas entre le niveau du micro-pouvoir et le niveau du macro-pouvoir de coupure et que quand on parle de l'un, on n'exclut pas de parler de l'autre. Une analyse en termes de micro-pouvoirs rejoint sans difficulté l'analyse de problèmes comme ceux du gouvernement et de l'État (Foucault 2004 : 366). Ainsi, dans le cas du Japon, on peut considérer que les idées et techniques qui contribuèrent à structurer la politique de gestion des populations ouvrières circulèrent dans des milieux qui ne furent pas seulement représentés par les hauts fonctionnaires, mais aussi dans des milieux de la réforme sociale appartenant non seulement aux milieux syndicaux, mais également au milieu émergeant des médias, au milieu scientifique ou au milieu des affaires qui constituaient ce que l'on peut appeler des micro-pouvoirs.

Par exemple, une des mutations majeures qui permettra la naissance des politiques de gestion moderne des populations ouvrières sera l'émancipation des réformateurs sociaux vis-à-vis de conceptions traditionnelles de l'assistance qui n'étaient plus du tout adaptées aux problèmes du Japon industriel qui prend alors forme. Ce travail, fondateur, de redéfinition des catégories de populations touchées par l'action des politiques publiques, se fera très largement au départ, non pas à l'initiative de l'État, mais de journalistes répondant à la curiosité de leur lectorat à l'égard de la question sociale qui s'affirme et qui réalisent les premières enquêtes.

Les conceptions anciennes sont encore visibles dans le "Règlement sur l'assistance "(Jukkyū kisoku 恤救規則) de 1874 qui organise la politique d'assistance sociale de l'État suite à la restauration de Meiji. Le préambule du règlement stipulait en effet d'emblée que celle-ci devait reposer sur une entraide populaire (jinmin sōgo no joggi 人民相互/情誼) et que ne pouvaient être secourus que les nécessiteux désaffiliés (mukoku no kyümin 無告)笨 民). Les cinq articles de la loi précisaient ces restrictions. N'étaient susceptibles de recevoir une aide que les très pauvres (gokuhin no mono 極貧/者) et 
célibataires (dokushin 独身), dans l'incapacité de travailler pour cause d'infirmité (haishitsu ni kakari sangyō o itonamu atawazaru mono 廃疾二罹り産業 営ム能ハサル者), les personnes de 70 ans ou plus dans l'incapacité de travailler parce que gravement malades ou séniles (shichijūnen ijō no mono jübyō aruiwa rōsui shite sangyōo itonamu atawazaru mono 七十年以上ノ者重病或八老衰シ テ産業习営ム能ハサル者), les personnes malades dans l'incapacité de travailler (shippei ni kakari sangyōo itonamu atawazaru mono 疾病二罹リ産業习営么能 ハサル者), et enfin les personnes âgées de 13 ans et moins (jüsannen ika no mono 十三年以下/者). Les non-célibataires ne pouvaient être secourus que si les autres membres de leur famille étaient dans une incapacité de travailler résultant du jeune âge, d'une infirmité liée au vieil âge, à la maladie, ou d'un handicap de naissance (Endō 1991 : 58-59).

Cependant, avec l'industrialisation, ces limitations issues de conceptions néo-confucéennes qui rejetaient les populations ouvrières en dehors du champ d'action du gouvernement, se retrouvent en complet décalage avec la réalité, car le pauvre devient surtout le pauvre qui travaille. Les premières enquêtes, à partir de la deuxième décennie de l'ère Meiji, se concentrent exclusivement sur les couches les plus pauvres et les plus déchues de la population, ayant en général été complètement exclues du monde du travail. Si ces "enquêtes sur les bas-fonds " (hinminkutsu tōsa 貧民窟踏査), souvent l'œuvre de journalistes, contribuent sans aucun doute à l'éveil des consciences, elles renvoient aussi à une image traditionnelle de la pauvreté, monde marginal. Un des plus grands succès de ce type de littérature fut par exemple l'ouvrage du journaliste Matsubara Iwagorō 松原岩五郎, Tokyo des plus profonds ténèbres (Saiankoku no Tokyo 最暗黒の東京, 1893) (Matsubara 1988 : 15-16). Néanmoins, au tournant du siècle, influencé notamment par les enquêtes menées en Grande Bretagne, un nouveau type d'enquêtes commence à appréhender la pauvreté de façon beaucoup plus large et à la lier à une question du travail. En plus des " miséreux" (hinmin 貧民) des bas fonds, sont aussi pris en compte les "démunis" (saimin 細民), embryons de la classe ouvrière. Le premier à conduire une telle enquête est Yokoyama Gennosuke 横山源之助, journaliste au quotidien Mainichi. C'est avec Les basses couches du Japon (Nihon no kasō shakai 日本之下層社会), publié en 1899, qu'il donne le ton à la monumentale enquête sur la condition des ouvriers - shokkō jijō 職工事情 - du ministère de l'Agriculture et du Commerce en 1903, et qui servira de base à la rédaction de la Loi sur les usines de 1911, et aux régulières " enquêtes sur les démunis " (saimin chōsa 細民調査), inaugurées en 1911, du Bureau des affaires locales du 
ministère de l'Intérieur (Naimushō Chihōkyoku 内務省地方局) (Nakagawa 1986 : 152-153, 158-159).

Cette influence de la société civile se vérifie aussi dans le cas des premières enquêtes sur le budget des ménages ouvriers. Les enquêtes sociales qui avaient pris leur essor à partir des années 1890 abordaient la question du budget des ménages, mais n'utilisaient pas de méthode statistique. C'est Takano Iwasaburō 高野岩三郎, membre de la Société pour l'étude de la politique sociale (Shakai seisaku gakkai 社会政策学会) ayant étudié en Allemagne les statistiques avec George von Mayer après avoir été diplômé de l'université de Tokyo en 1895, qui introduisit une telle application des statistiques aux enquêtes sociales. Takano était devenu membre du Yūaikai 友愛会, le premier syndicat ouvrier d'envergure au Japon, dès sa création en 1912. C'est grâce à son soutien qu'il avait pu réaliser, en mai 1916, une enquête sur le budget familial de 20 ménages d'ouvriers de Tokyo, membres du syndicat ${ }^{7}$. Et c'est grâce au succès de cette première expérience d'initiative privée que le Bureau de l'hygiène du ministère de l'Intérieur (Naimushō Eiseikyoku 内務省衛生 局) lui confia une enquête sur les budgets de 40 ménages ouvriers dans le quartier de Tsukishima 月島. Cette enquête donnera l'impulsion aux enquêtes régulières du ministère de l'Intérieur à partir des années 1920 (Shimamura 2008 : 139-142).

Deuxièmement, il faut prendre conscience que dépasser l'État comme unique horizon de la gestion des populations, c'est aussi dépasser les frontières strictes de la nation. La dynamique historique du Japon d'avant-guerre ne peut être réduite à un processus d'évolution s'étant nourri de sa propre logique, que cette logique ait été impulsée par la persistance d'éléments féodaux - incarnés au niveau politique par le système impérial - comme l'ont analysé les auteurs marxistes du Kōza-ha, ou par l'adoption initiale du modèle allemand dans la deuxième moitié de l'ère Meiji ${ }^{8}$. Les orientations prises par

7. Les résultats de cette enquête furent rendus publics sous le titre « Enquête sur le budget de 20 ménages ouvriers à Tokyo " (Tokyo ni okeru 20 shokkō kakei chōsa 東京ニ於ケル二 十職工家計調査) au sein d'un ouvrage consacré à la politique sociale en l'honneur de Kanai Noburu 金井延 (Kanai kyōju zaishoku 25 nen kinen. Saikin shakai seisaku 金井延教授在職 25年記念一一最近社会政策).

8. Erik Grimmer-Solem a récemment reproché à certains travaux (il cite en particulier les travaux de Kenneth Pyle et de Bernd Martin) d'avoir été victimes d'une vision téléologique 
les gouvernants pour construire la politique sociale japonaise ont été en effet successivement inspirées par des idées qui circulaient d'un continent à l'autre grâce aux contacts que pouvaient avoir entre eux les réformateurs sociaux audelà des frontières de leur propre pays.

Il faut d'ailleurs ici prendre acte du fait que l'historiographie japonaise a beaucoup changé depuis une dizaine d'années. Comme le souligne Pierre Souyri dans la revue Débat, la nouvelle génération d'historiens japonais a tendance à montrer la grammaire commune de la modernité qu'il a pu y avoir entre les pays occidentaux et le Japon (Souyri 2009). Il s'agit de faire un autre usage du comparatisme en montrant que les ressemblances avec l'expérience occidentale l'emportent sur les différences, mais aussi de faire de l'histoire connectée et de réhabiliter les facteurs transnationaux. L'étude d'une organisation dédiée à la réforme sociale telle que la Kyōchōkai illustre à merveille ce phénomène. Pourtant, comme nous l'avons déjà souligné auparavant, elle n'est souvent même pas citée dans les travaux spécifiquement consacrés à l'histoire de la politique sociale jusque dans les années 1980. Son étude a été en effet jugée comme à la fois moralement illégitime et scientifiquement de peu d'importance. Ou bien encore il en est fait mention, mais uniquement pour en souligner la fonction de simple instrument du patronat pour contrer l'idée de lutte des classes par une rhétorique de coopération entre le capital et le travail et détruire ainsi le mouvement syndical qui prenait alors de l'ampleur. Par exemple, le premier des trois volumes qui furent publiés en 1970 par le Groupe de recherche sur le fascisme de l'université de Waseda - une des productions majeures de l'historiographie marxiste sur le fascisme japonais - n'en fait qu'un simple supplétif du capital monopolistique d'État dans le mécanisme de mise en place d'un "pré-fascisme " dans ces années 1920 (Waseda daigaku shakai kagaku kenkyūjo fashizumu kenkyūkai 1970 : 324327). Pourtant, le travail pionnier de Dean Kinzley (Kinzley 1991), suivi de travaux japonais plus récents (Takahashi 2001), en ont au contraire montré

de l'histoire moderne et contemporaine japonaise et d'avoir artificiellement plaqué la thèse du "Sonderweg " allemand sur l'histoire du réformisme social japonais. Ces travaux auraient attribué à l'intérêt porté au modèle allemand par les bureaucrates japonais - qui se détournent alors du libéralisme ayant inspiré les premières réformes socio-économiques de l'ère Meiji - le développement d'une éthique sociale collectiviste qui aurait détruit les germes de pluralisme et de démocratie et aurait finalement conduit à un totalitarisme qui prit toute son ampleur à partir du début des années 1930 (Grimmer-Solem 2005 : 187222 ; Pyle 1974 : 127-164 ; Martin 1995). 
l'importance pour comprendre les relations que purent entretenir une partie des élites dirigeantes japonaises avec l'internationale de la réforme sociale.

L'action de la Kyōchōkai dans le domaine de la politique sociale doit être comprise dans le cadre des rapports que le Japon entretient avec l'Organisation internationale du Travail. Les principaux fondateurs de la Kyōchōkai, le ministre de l'Intérieur, Tokonami Takejirō 床次竹二郎 et l'industriel Shibusawa Eiichi 啮沢栄一, son premier vice-président, étaient en effet convaincus que, en tant que première nation non occidentale à avoir été admise dans le club des grands, leur pays avait certaines responsabilités quant à l'avancement du progrès social (Takahashi 2001). D'autre part, si sa participation à la SDN et son adhésion à l'OIT lui donnaient pour la première fois de son histoire une place au sein des puissances de premier rang ${ }^{9}$, elle devait en retour respecter un certain nombre de normes sociales que le Bureau international du Travail s'appliqua à formuler tout au long des années 1920 et $1930^{10}$. L'importance que donna le gouvernement japonais à son adhésion est par exemple visible dans le fait que lors de la conférence internationale de Genève en 1920, le Japon envoya une délégation de 60 personnes, soit la plus importante. Il ne manqua aucune conférence jusqu'à ce qu'il quitte l'organisation en 1938.

Une des conséquences majeures de cette adhésion fut de diversifier la source des influences dans le domaine de la politique sociale et le sens que le pouvoir japonais donnait à cette dernière. Ainsi, si l'on regarde les sommaires du Bulletin de politique sociale (Shakai seisaku jihō 社会政策時報) - revue de la Kyōchōkai -, tout au long des années 1920, ce sont les législations sociales et les idées de réforme sociale surtout en provenance de Grande Bretagne, des États-Unis ou de France qui viennent s'ajouter aux références plus traditionnelles du modèle social allemand. Par exemple le solidarisme français, le fabianisme britannique ou bien encore les politiques paternalistes des grandes entreprises américaines. Parallèlement à la technologie de gestion de la santé des ouvriers, que représente l'hygiénisme industriel d'origine allemande, on

9. Elle lui permettait en parliculier de défendre ses intérêts face à l'accusation de la Grande Bretagne de dumping social qui se prolongea tout au long des années 1920-1930.

10. Cette influence, directe ou indirecte, est bien mis en avant par le rapport d'Ayuzawa Iwao 鮎沢篇, chef du bureau de Tokyo du BIT. Ce rapport ne sera néanmoins pas publié suite au départ du Japon de l'Organisation internationale du Travail en 1938. Un exemplaire se trouve conservé à l'université de Columbia, dans les archives de Lindsay Roger, ancien directeur adjoint du BIT. Je remercie Thomas Cayet de l'avoir porté à ma connaissance (Ayuzawa 1938). 
voit en particulier apparaître des articles défendant la légalisation des syndicats qui permettraient aux travailleurs d'être les acteurs de leur propre intégration sociale. S'il ne parviendra pas à faire adopter une loi protégeant l'activité des syndicats, le gouvernement japonais reconnaîtra de facto leur légitimité quand son adhésion à l'OIT l'obligera à envoyer des représentants du travail issus des organisations de travailleurs existantes.

Bien sûr, après le début de la crise et l'affaire de la Mandchourie en 1932, on voit un déplacement assez violent des modèles. Dans le Bulletin de politique sociale, on ne compte plus, à partir de 1933, les articles sur les politiques économiques et sociales nazie et fasciste. La modernisation de la gestion des populations ouvrières devient très largement synonyme d'introduction de nouvelles techniques visant à augmenter le niveau de mobilisation spirituelle et les capacités physiques des travailleurs, au moyen notamment de la diététique, de l'exercice physique ou de l'eugénisme. Cependant, cette évolution des politiques de gestion des populations ouvrières au Japon ne se réduit pas à un changement de modèle, c'est l'ensemble des modèles eux-mêmes qui changent. Par exemple, l'évolution de l'idée que la Kyōchōkai se fait de la politique sociale évolue parallèlement avec les conceptions du Bureau international du Travail lui-même. L'évolution des sommaires du Shakai seisaku jihō, font très largement écho à l'évolution des sommaires de la Revue internationale $d u$ travail, principale revue du BIT, qui se ne préoccupe plus exclusivement de la question de l'amélioration des conditions de travail, mais de plus en plus, dans sa volonté d'aborder la question du chômage à sa source, de politique économique et même de politique démographique. Voilà ce qu'écrit dans le Shakai seisaku jihō en juillet 1932, Yoshida Shigeru 吉田茂, le directeur de la Kyōchōkai :

Il est difficile de mettre en place en un jour une politique sociale efficace qui pourrait relever les défis actuels. Mais en même temps il est difficile de s'en tenir à la politique qui a été menée jusqu'à aujourd'hui... Ce qu'il ne faut pas oublier aujourd'hui, c'est la relation intime qu'entretient la politique sociale avec les autres politiques. Dans notre pays, la politique économique et la politique sociale n'ont-elles pas le même but ? Le problème social le plus important de notre pays aujourd'hui est le chômage, et si l'on s'interroge sur ce que devrait être une politique efficace en mesure d'aider les chômeurs, il y est évident que la mission de la politique sociale est de réformer les politiques qui concernent l'économie industrielle ou la population. (Yoshida 1932 : 1-4) [notre traduction].

Le passage suivant d'un numéro de la Revue internationale du travail montre que, loin de traduire une prise de distance de la Kyōchōkai avec les orienta- 
tions libérales du Bureau international, le discours de Yoshida Shigeru illustre une évolution en grande partie parallèle :

La crise économique met en évidence la précarité du progrès accompli tant que le travailleur reste exposé à perdre un jour ou l'autre toute possibilité d'emploi. Le chômage devient le souci principal de tout gouvernement et lui fait adopter une attitude nouvelle à l'égard des problèmes économiques. Il fait entrer ces problèmes dans les préoccupations de la politique sociale et oriente vers le progrès social la politique économique. Désormais, tout en continuant à poursuivre la solution des problèmes qui constituent son domaine propre, la politique sociale tend à influencer la politique économique dans un sens qui lui permettra, facilitera ou consolidera son progrès [...] on a pu constater que la grande crise économique des dernières années, parmi tant d'autres résultats révolutionnaires, aura eu celui de briser définitivement la barrière, que certains dogmatiques avaient dressée et voulait maintenir, entre l'économique et le social. (Revue internationale du travail $1939: 316)$.

Ainsi, on peut dire que si le mur entre politique sociale et politique économique s'effondre et que la politique de gestion de la population est de plus en plus envisagée aussi au moyen de techniques de pilotage de l'économie, ce n'est pas à cause seulement d'une évolution interne au Japon. C'est d'une part aussi parce que les yeux des experts se tournent vers les pays qui posent cette nouvelle problématique de la façon la plus radicale, mais aussi, d'autre part, parce que cette nouvelle problématique se pose dans tous les pays industrialisés de cette époque.

Enfin, troisièmement, il faut avoir conscience que s'il faut sortir des visions trop culturalistes ou essentialistes de l'histoire du Japon d'avant-guerre, il ne faut pas non plus réduire le processus de modernisation à un processus d'acculturation. Si la circulation transnationale des idées de la réforme sociale et des techniques de gestion de la population a très largement atteint le Japon, et que ce dernier a très naturellement été influencé par un contexte international qui s'appliquait à tous les pays, il n'en a pas moins aussi développé des solutions qui lui étaient propres. Nous reprendrons ainsi à notre compte la formule de Jack Goody selon laquelle il s'agit « à la fois de briser le compartimentage mutilant des frontières nationales et de sortir du "grand récit" de l'occidentalisation de la planète : l'histoire du monde ne saurait être réduite à l'ascension de l'Ouest et à l'occidentalisation du reste "(Goody 2007). La modernisation des techniques de gestion des populations ouvrières n'a pas été l'histoire d'un simple transfert au Japon de modèles nés en Occident. Les réformateurs sociaux japonais ont opéré un tri parmi les solutions qui leur venaient des autres pays industrialisés en fonction des objectifs qu'ils estimaient prioritaires 
pour leur pays et, en même temps, ont continué à être marqués par des représentations sociales néo-confucéennes issues de la période pré-moderne, ou réinventées, en réaction à l'introduction d'idéologies étrangères, pour nourrir la construction du nouvel ordre social.

Le cas de la mise en place des dispositifs d'assistance aux populations vulnérables, les hōmen iin seido 方面委員制度 (système d'assistants sociaux de quartier) qui, avec le passage de la Loi sur l'assistance (Kȳ̄go hō 救護法) de 1929, devint un des dispositifs centraux du système d'assistance sociale est tout à fait révélateur. Ce système fut introduit à la fin de la première guerre mondiale en provenance d'Allemagne, mais correspondait aussi à une orientation de la politique sociale japonaise beaucoup plus ancienne. Introduit en 1917 à Okayama à l'initiative du préfet Kasai Shin.ichi 笠井信一, sous le nom de Okayama-ken saisei komon (seido) 岡山県済世顧問制度 (conseiller de réforme sociale de la préfecture d'Okayama) puis en 1919 par le préfet Hayashi Ichizō 林一蔵, et le réformateur social Ogawa Shigejirō 小河滋次郎, sous le nom de hōmen iin seido à Osaka, ce système d'assistants sociaux bénévoles était pensé comme un moyen de soulager et de prévenir la pauvreté de la classe ouvrière au travers de la bienfaisance des classes moyennes. Il utilisait le système allemand Elberfeld comme modèle, un système baptisé du nom de la ville où il fut codifié en 1853 et d'où il se répandit dans les principales villes d'Allemagne jusqu'en 1914 et dans un certain nombre de localités en Angleterre (Tipton 2008 : 365). Néanmoins, réduire son introduction au Japon à une simple importation de l'étranger est réducteur. Comme ceux qui souhaitaient son introduction en Angleterre, les autorités japonaises étaient attirées par le system Elberfeld parce qu'il était compatible avec ce qu'ils appelaient les "belles traditions " japonaises d'entraide mutuelle et notamment locales. Naitō Yoshihiro 内藤義弘 les présentait ainsi en 1929 dans le Shakai seisaku jihō :

Au Japon, il existe dans le système social un fort système familial et une tradition d'entraide. Ainsi, le travailleur, même s'il est au chômage ne se trouvera pas tout de suite dans l'embarras. Il peut solliciter un parent, un aîné de son village ou de son quartier ou un de ses professeurs d'école pour trouver un emploi. Lorsqu'on ne retrouve pas rapidement le chemin de l'emploi, il pourra être nourri ou même logé chez de telles personnes. Au Japon, cela n'est pas seulement le cas chez les riches, mais aussi dans les familles de classes moyennes ou de travailleurs. C'est là une belle coutume [ryōfü bizoku 良風美俗]. (Naitō $1929: 2$ ) [notre traduction].

Cette volonté de favoriser les solidarités de proximité était déjà centrale dans le règlement sur l'assistance de 1874. Et si elles étaient centrales dans le 
règlement de 1874, c'est qu'elles étaient au centre des conceptions sur l'assistance développées par des penseurs néo-confucéens comme Ōgyū Sorai 荻生徂徠, Ninomiya Sontoku 二宮尊徳 ou Yamaga Sokō 山鹿素行 (Ikeda 1986 : 94). En effet, pour limiter les dépenses publiques et encourager les "solidarités primaires ", Yamaga Sokō, un des penseurs confucéens de l'assistance aux pauvres les plus en vue de son époque, estimait qu'il fallait avant tout privilégier le soutien familial (shinzoku no yashinai 親族の養い). Si le nécessiteux était sans famille, il fallait que ce soit le village dans son ensemble (isson ichigo 一村一鄉) qui prodigue l'assistance. C'est seulement si le village lui-même était trop pauvre qu'un fonctionnaire devait étudier la "faisabilité d'une assistance par le haut " (Ikeda 1986 : 95).

\section{Conclusion}

Au Japon aussi, dès la période d'avant-guerre, le domaine de la gestion des populations s'est imposé véritablement comme une nouvelle forme de gouvernance, caractéristique du processus de modernisation des États industriels. Pour pouvoir le montrer sans ambiguïtés, nous avons vu qu'il était important d'avoir trois soucis qui ont été longtemps négligés : ne pas réduire les politiques de gestion des populations ouvrières à un processus d'imposition par le haut - par l'État - de mesures de contrôle social ; dépasser les frontières strictes de la nation dans le développement de la politique sociale au Japon pour sortir des visions trop culturalistes ou essentialistes de l'histoire japonaise d'avant-guerre ; mais ne pas réduire non plus le processus de modernisation à un processus d'acculturation.

C'est à ces conditions que l'on pourra efficacement combattre l'idée fausse d'un système socio-économique japonais encore très largement arriéré avant 1945, et à l'écart des grands mouvements de réformes sociales des pays industrialisés de l'époque, et que l'on pourra ainsi à nouveau « historiciser » ou bien encore " historiciser " autrement le Japon d'avant-guerre. L'" historiciser " autrement en tentant de s'extraire des grandes controverses, comme celle ayant opposée Ōkōchi et Kazahaya, qui furent déterminées par des enjeux politiques qui eurent tendance à étouffer le débat scientifique. L' « historiciser " à nouveau face aux interprétations substantialistes et culturalistes qui n'ont cessé de voir le jour au cours de l'après-guerre. Une telle tendance est par exemple bien illustrée par le classique de Ruth Benedict The Chrysanthemum and the Sword, écrit pendant la guerre pour l'Office of War Information de Washington dans 
le but de renseigner les militaires sur l'identité de leur ennemi, et qui était la version haut de gamme de la vulgate culturaliste qui contribuait à façonner les représentations du Japon qui habitaient les occupants américains. Ils comprenaient en effet les institutions et l'idéologie économiques et sociales sur lesquelles reposaient le régime impérial d'avant 1945 comme, si l'on grossit un peu le trait, l'expression d'une structure anthropologique immuable, traduit sur le plan politique par un obscurantisme " néo-féodal " et qui aurait finalement facilité une évolution de type fasciste (Benedict 1946). Si les études savantes traitant du système social japonais au $\mathrm{xx}^{\mathrm{e}}$ siècle ont bien sûr parcouru beaucoup de chemin depuis, il est sans doute encore nécessaire de lutter contre un " orientalisme ", dans lequel l'historiographie japonaise a d'ailleurs une part de responsabilité, et qui continue encore à imprégner, envers et contre tout, nombre de représentations que se forgent les sociétés occidentales sur le Japon, et notamment sur le rapport que celui-ci a pu entretenir avec la modernité.

\section{Bibliographie}

\section{AYUZAWA Iwao, 1938}

Japan and the ILO: A Survey of the Influence of the ILO on Industrial and Labour

Conditions in Japan during 1919-1938,

Tokyo Office, International Labour Office, December.

\section{BENEDICT Ruth, 1946}

The Chrysanthemum and the Sword, Boston, Houghton Mifflin.

\section{ENDŌ Kōichi 遠藤興一, 1991}

Shiryō de tsuzuru shakai fukushi no ayumi 史料でつづる社会福祉の歩み (La marche du bien-être social vue par les documents historiques), Tokyo, Fumaidō shuppan 不昧堂出版.

\section{FOUCAULT Michel, 2004}

Sécurité, territoire, population : Cours au

Collège de France, 1977-1978, Paris,

Gallimard Seuil.

\section{GOODY Jack, 2007}

The Theft of History, Cambridge, Cambridge University Press.

\section{GRIMMER-SOLEM Erik, 2005}

« German Social Science, Meiji

Conservatism, and the Peculiarities of Japanese History ", Journal of World History, 16 (2) : 187-222. 
IKEDA Yoshimasa 池田敬正, 1986

Nihon shakai fukushi shi 日本の社会福祉

史 (Histoire du bien-être social au Japon), Tokyo, Hōritsu bunkasha 法律文化社.

KAZAHAYA Yasoji 風早八十二, 1937

Nihon shakai seisaku shi 日本社会政策史 (Histoire de la politique sociale au Japon), Tokyo, Nihon hyōronsha 日本評論社.

\section{KINZLEY D.W., 1991}

Industrial Harmony in Japan

- The Invention of a Tradition, London,

Routledge.

KISHIMOTO Eitarō 岸本英太郎, 1950 Shakaiseisakuron no kompon mondai 社会政策論の根本問題 (Les enjeux fondamentaux du débat sur la politique sociale), Tokyo, Nihon hyōron shinsha 日本評論新社.

\section{MARTIN Bernd, 1995}

Germany and Japan in the Modern World, Providence, Berghahn Books.

MATSUBARA Iwagoro 松原岩五郎, 1988 [rééd.]

Saiankoku no Tokyo 最暗黒の東京 (Tokyo des plus profonds ténèbres), Tokyo, Iwanami bunko 岩波文庫.

\section{NAITŌ Hisahiro 内藤義弘, 1929}

« Wagakuni ni okeru shitsugyō mondai » 我が国に於ける失業問題 (La question du chômage dans notre pays), Shakaiseisaku jihō 社会政策時報, 108:38-59, septembre.

NAKAGAWA Kiyoshi 中川清, 1986

Nihon no toshi kasō 日本の都市下層 (Les basses couches urbaines), Tokyo, Keisō shobō 勁草書房.
ŌKŌCHI Kazuo 大河内一男, 1939

«Gainen kōsei o tsūjite mitaru shakai seisaku no hensen » 概念構成を通じて見 たる社会政策の変遷 (Les évolutions des conceptions de la politique sociale), Keizaigaku ronshū 経済学論集, shinkan dai $9 \mathrm{gō}$ 新刊第9号 : 26-40.

\section{ŌKŌCHI Kazuo, 1940a}

Shakai seisaku no kihon mondai 社会政策 の基本問題 (Les questions fondamentales de la politique sociale), Nihon hyōronsha 日本評論社.

\section{ŌKŌCHI Kazuo, 1940b}

Senji shakai seisaku ron 戦時社会政策論 (La politique sociale du temps de guerre), Tokyo, Jichōsha 時潮社.

\section{ŌKŌCHI Kazuo, 1968}

Ōkōchi Kazuo chōsakushū 大河内一男著作集, Tokyo, Seirin shoin shinsha 青林書院新社, vol. 1.

\section{ŌKŌCHI Kazuo, 1970}

Shakaiseisaku 40 nen 社会政策四十年 (40 ans de politique sociale), Tokyo, Tokyo daigaku shuppankai 東京大学出版会.

\section{PYLE Kenneth, 1974}

«Advantages of Followership : German Economics and Japanese Bureaucrats, 1890-1925 », Journal of Japanese Studies, 1 (1) : 127-164.

Revue Internationale du travail, 1939 Numéro spécial , "Politique sociale et population », 39 (3), mars.

SHIMAMURA Shirō 島村史郎, 2008 Nihon tōkei hattatsushi 日本統計発達 史 (Histoire du développement des 
statistiques au Japon), Tokyo, Nihon tōkei kyōkai 日本統計協会.

SOUYRI Pierre-François, 2009

« La modernité japonaise dans tous ses

états », Le Débat, 153 : 88-99.

TAKAHASHI Hirohiko 高橋彦博, 2001

Senkanki Nihon no shakai kenkyū sentāŌhara shaken to Kyōchōkai 戦間期日本の 社会研究センター一大原社研と協調会 (Les centres de recherche sur la société du Japon de l'entre-deux-guerres. Le Centre de recherches Ōhara sur la question sociale et la Société pour la coopération), Tokyo, Hakushobō 柏書房.

TIPTON Elise K., 2008

" Defining the Poor in Early Twentiethcentury Japan », Japan Forum 20 (3).

Waseda daigaku shakai kagaku

kenkyūjo fashizumu kenkyūkai 早稲田大 学社会科学研究所 ファシズム研究部会, 1970 Nihon no fashizumu I : keiseiki no kenkyû 日本のファシズム 第 1 巻 形成期の研究 (Le fascisme du Japon, vol. 1 : Recherches sur la période de formation), Tokyo, Waseda daigaku shuppanbu 東京早稲田大学出版部.

YOSHIDA Shigeru 吉田茂, 1932

《Shakaiseisaku no saikentō » 社会政策の 再検討, Shakai seisaku jihō 社会政策時報， $146: 1-3$. 